\title{
One year experience using mycobacterial blood cultures to diagnose tuberculosis in patients with prolonged fever in Vietnam
}

\author{
Diep NT Nguyen ${ }^{1}$, Trung V Nguyen ${ }^{2}$, Trinh T Dao ${ }^{2}$, Lam T Nguyen ${ }^{2}$, Peter Horby $^{1,3}$, Kinh V Nguyen ${ }^{2}$, \\ Heiman FL Wertheim ${ }^{1,3}$ \\ ${ }^{1}$ Wellcome Trust Major Overseas Program Vietnam, Oxford University Clinical Research Unit (OUCRU), Hanoi, \\ Vietnam \\ ${ }^{2}$ National Hospital for Tropical Diseases (NHTD), Hanoi, Vietnam \\ ${ }^{3}$ Centre for Tropical Medicine, Nuffield Department of Clinical Medicine, University of Oxford, Oxford, United \\ Kingdom
}

\begin{abstract}
Introduction: To evaluate the use of mycobacterial blood cultures (MBC) in diagnosing tuberculosis (TB) in patients with prolonged fever admitted to a Vietnamese referral hospital.

Results: MBCs from 94 patients (66\% male; median age 33 years; 75\% HIV positive) were evaluated: 14 were mycobacterium positive (all HIV positive), and MBC was the only positive specimen in 9 cases (41\%). Three positive cases were identified as Mycobacterium avium and the remaining M. tuberculosis (one case could not be identified).

Conclusion: $\mathrm{MBC}$ can be a valuable additional method to diagnose TB, particularly in immunosuppressed HIV patients when sputum cannot be collected.
\end{abstract}

Key words: tuberculosis; blood culture; HIV.

J Infect Dev Ctries 2014; 8(12):1620-1624. doi:10.3855/jidc.4895

(Received 21 February 2014 - Accepted 28 May 2014)

Copyright $(2014$ Nguyen et al. This is an open-access article distributed under the Creative Commons Attribution License, which permits unrestricted use, distribution, and reproduction in any medium, provided the original work is properly cited.

\section{Introduction}

The Wolrd Health Organization estimated that 1.7 million people died of tuberculosis (TB) in 2009, including 1.3 million deaths in HIV-negative people and 0.4 million deaths in HIV-positive people. Vietnam is one of 22 countries with the highest burden of TB, where approximately 32,000 people died from TB in 2009 and where multi-drug resistant TB (MDRTB) is emerging [1]. It is important to have adequate TB diagnostics for both patient treatment and disease control. However, the diagnosis of tuberculosis can be challenging, especially in developing countries [2-4].

Several methods of TB diagnosis are commonly used worldwide, including: acid-fast bacilli (AFB) smear, culture of sputum on solid or in liquid medium, and molecular diagnostics [5,6]. HIV-infected patients with suspected pulmonary TB often lack a productive cough, thus sputum is difficult to collect [7]. Furthermore, the rate of sputum smear negativity is relatively high, ranging from $24 \%$ to $61 \%$ [8-10]. Patients with extra-pulmonary $\mathrm{TB}$ usually require invasive specimen collection procedures for $\mathrm{TB}$ diagnosis $[11,12]$. In such cases, blood culture for TB may help to confirm the diagnosis.

Mycobacterial blood cultures have shown varying diagnostic yields. One Brazilian study found mycobacteremia in $60 \%$ of 33 tuberculosis infected HIV patients and blood was the only positive specimen in 5 patients (15\%) [13]. Another study in Spain showed that blood was the only positive sample in 33\% of tested patients [14]. In this study we present the results of a one year observational study on the use of blood cultures to diagnose $\mathrm{TB}$ in patients with prolonged fever in northern Vietnam.

\section{Methodology}

Study population and specimen collection

This is a descriptive study on the routine clinical usage of mycobacterial blood cultures in patients admitted to the National Hospital for Tropical Diseases (NHTD) between September 2009 to September 2010 with prolonged fever of unknown origin. Patients diagnosed with prolonged fever where defined as those with more than two weeks of 
unexplained fever. Specimens were obtained at the discretion of the treating physician: bacterial and fungal blood cultures, sputum for AFB smear and TB PCR, cerebrospinal fluid (CSF), and aspirates (e.g. pleural fluid). All data were collected and stored anonymously for this descriptive study. This study was considered to be an evaluation of medical services by the Ethical Review Board of NHTD and the need for informed consent was therefore waived. In Vietnam the standard work up for TB is performing a sputum acid fast smear in suspect patients. Suspect patient are considered those with prolonged productive cough $(>2$ weeks). In case of HIV in the absence of productive cough, patients are treated empirically when TB is suspected. Induced sputum is generally not done as facilities to do this safely are not available.

\section{Mycobacterial diagnostics}

Sputum specimens were pre-processed using BBL MycoPrep Specimen Digestion/Decontamination Kit (Becton Dickinson, Franklin Lakes, USA) according to manufacturer's instructions. The sediment was stained by Ziehl-Neelsen (ZN) to detect AFB and graded according to WHO recommendations by microscopy [15].

For mycobacterial blood cultures, $5 \mathrm{ml}$ of blood was collected in Myco/F lytic (MFL) Bactec bottles (Becton Dickinson, USA) and incubated in the Bactec 9050 instrument for 42 days. All positive MFL cultures were removed and AFB smear performed. MFL cultures that gave a positive signal within 42 days but did not contain AFB were returned into the instrument. MFL cultures positive with AFB were further tested for TB identification and drug resistance by GenoType MTBDRplus assay (HainLifesciences, Nehren, Germany). If MFL cultures were negative with GenoType MTBDRplus assay, Mycobacterium avium complex (MAC) PCR was performed, as described previously [16]. Routine TB PCR at our hospital is ordered at the discretion of the treating doctor and has IS6110 insertion sequence as target as described elsewhere [17].

\section{Routine bacterial and fungal blood culture}

Routine bacterial and fungal blood cultures were performed by Aerobic F Bactec (AF) bottles (Becton Dickinson, USA). In case of growth, bacteria or fungi were further identified using standard microbiological techniques.

\section{Results}

Patients with mycobacterial blood culture performed.

During the one-year evaluation period, mycobacterial blood cultures were performed for 94 patients. Seventy patients $(75 \%)$ were HIV positive, with relatively more men: $82 \%$. All of the patients from which mycobacterial blood cultures were collected had signs suggestive of tuberculosis, including: prolonged fever of unknown origin, weight loss, cough, and fatigue. Fever lasted for a median of 27 days (IQR: 12-33.5) before blood for mycobacterial culture was collected. The characteristics of enrolled patients are summarized in Table 1 .

From the 94 evaluated patients, AFB in any specimen was detected in 22 patients: blood cultures alone $(n=9)$, sputum smear alone $(n=5)$, PCR alone $(\mathrm{n}=3)$, combination of blood culture and sputum smear $(n=4)$, combination of blood cultures and PCR $(n=1)$. A total of fourteen $(15 \%)$ mycobacterial blood cultures were positive and all were from HIV-infected patients (Table 2). From these 14 cases, 13 strains were tested by the MTBDRplus assay (one specimen was accidentally discarded before testing). Ten patients were mycobacterial positive with this assay: 6

Table 1. Characteristics of 94 patients with prolonged fever of unknown origin.

\begin{tabular}{lccc}
\hline Characteristics & $\begin{array}{c}\text { All patients } \\
(\mathbf{n}=\mathbf{9 4})\end{array}$ & $\begin{array}{c}\text { Positive Mycobacterial Blood } \\
\text { culture } \\
(\mathbf{n = 1 4 )}\end{array}$ & $\begin{array}{c}\text { Negative Mycobacterial Blood } \\
\text { culture } \\
(\mathbf{n}=\mathbf{8 0})\end{array}$ \\
\hline Age, median (IQR) & $33(30-39)$ & $31(30-33)$ & $34(30-40)$ \\
Male sex & $62(66 \%)$ & $11(79 \%)$ & $51(64 \%)$ \\
Fever & $94(100 \%)$ & $14(100 \%)$ & $80(100 \%)$ \\
Cough & $31(33 \%)$ & $4(29 \%)$ & $27(34 \%)$ \\
Weight loss & $33(35 \%)$ & $5(36 \%)$ & $28(35 \%)$ \\
Fatigue & $15(16 \%)$ & $5(36 \%)$ & $10(13 \%)$ \\
HIV positive & $70(75 \%)$ & $14(100 \%)$ & $56(70 \%)$ \\
Hospitalization days & $13(8-22)$ & $16(9-30)$ & $12.5(8-20)$ \\
Median (interquartile range) & & \\
\hline
\end{tabular}


patients were susceptible with both rifampicin (RMP) and isoniazid (INH), 2 patients were resistant to both RMP and INH, 1 patient was resistant to RMP only, and 1 patient was resistant to INH only. Three cases that were negative with the MTBDRplus assay were all positive with $M$. avium by MAC PCR. The median time to positivity for mycobacterial blood cultures was 24.5 days (IQR: 20-27).

The CD4 counts for 50/70 HIV-infected patients were known and analyzed. Those who were mycobacterial blood culture positive never had CD4 counts exceeding 100 cells $/ \mathrm{mm}^{3}$. The mean CD4 count for mycobacterial blood culture positive patients was lower than for negative patients: 42.1 (95\% CI: 21.3 62.9) versus 66.3 (95\% CI: 40.3- 92.2) respectively, not significant. For 52/70 HIV-infected patients we had data on anti-retroviral therapy (ART). Generally those that were already on ART at admission had lower mycobacteremia rates as compared to those not on ART: $1 / 19(5.3 \%)$ versus 9/33 (27.3\%). The remaining four mycobacteremia cases we had no data on ART.

Sputum was collected from 61 patients for AFB smear. The other 33 patients did not submit sputum samples, generally due to non-productive cough. Nine $(15 \%)$ of 61 patients were AFB positive. From 12 patients TB PCR was requested and performed for the following specimens: $\operatorname{CSF}(\mathrm{n}=9)$, sputum $(\mathrm{n}=1)$, abdominal fluid $(\mathrm{n}=1)$ and pleural fluid $(\mathrm{n}=1)$. From these 12 patients, 4 patients were positive with tuberculosis (2 CSF, 1 abdominal fluid, 1 sputum). AF blood cultures bottles were collected from 87 patients to detect other bacterial and fungal pathogens. Of those, 19 patients were positive and Penicillium marneffei was the most commonly identified pathogen.

The median time of hospitalization of enrolled patients was 13 days (IQR: 8-22). Most patients were discharged before the result of MFL culture was available. Eight of the mycobacterial blood culture positive patients were transferred to a tuberculosis hospital for treatment. From the remaining six: four died, one went home and was readmitted to a TB hospital due to positive mycobacterial blood culture, and one was lost to follow up. One MAC positive case with follow-up data received MAC treatment when the result became known.

\section{Discussion}

In this study, a total of 22 out of 94 cases were diagnosed with a mycobacterial infection (any specimen) by all methods of which 14 cases $(64 \%)$ were blood culture positive. From the 14 blood cultures positive cases, 9 cases were positive by blood culture only. Hence, blood was the only positive specimen in $41 \%$ of mycobacteria infected cases. Our positive rate of blood cultures was much higher than positive blood cultures rates reported in another study conducted in Vietnam, Thailand and Cambodia [18]. This study performed mycobacterial blood cultures on HIV-infected outpatients, including those with no particular symptoms like fever, and therefore this population was less likely to have TB than our population [18].

AFB smear detected 9 cases of all 22 mycobacterial cases in our study. Other studies have

Table 2. Summary of 14 positive - TB blood cultures (MFL) from National Hospital of Tropical Diseases, September 2009September 2010.

\begin{tabular}{|c|c|c|c|c|c|c|c|c|c|}
\hline Patient & $\begin{array}{l}\text { MFL Blood } \\
\text { culture }\end{array}$ & TB PCR & $\begin{array}{c}\text { AF blood } \\
\text { culture }\end{array}$ & $\begin{array}{c}\text { Sputum } \\
\text { AFB smear }\end{array}$ & $\begin{array}{c}\text { Time to } \\
\text { positivity } \\
\text { (days)* }\end{array}$ & $\begin{array}{c}\text { Identificati } \\
\text { on }\end{array}$ & RMP & INH & Outcome \\
\hline 1 & Pos & ND & Neg & Pos & 15 & M.avium & ND & ND & Survived \\
\hline 2 & Pos & Neg & Neg & Pos & 21 & MTB & $\mathrm{R}$ & $\mathrm{S}$ & Died \\
\hline 3 & Pos & Neg & $\mathrm{Neg}$ & Neg & 35 & MTB & $\mathrm{R}$ & $\mathrm{R}$ & Survived \\
\hline 4 & Pos & ND & Neg & Neg & 33 & MTB & $\mathrm{S}$ & $\mathrm{S}$ & Survived \\
\hline 5 & Pos & Neg & Neg & ND & 27 & MTB & $\mathrm{R}$ & $\mathrm{R}$ & Died \\
\hline 6 & Pos & Pos & $\mathrm{Neg}$ & ND & 34 & MTB & $\mathrm{S}$ & $\mathrm{S}$ & Died \\
\hline 7 & Pos & ND & Neg & Neg & 34 & M.avium & ND & ND & Survived \\
\hline 8 & Pos & Neg & Neg & Neg & 20 & MTB & $\mathrm{S}$ & $\mathrm{S}$ & Survived \\
\hline 9 & Pos & ND & Neg & $\mathrm{Neg}$ & 20 & M.avium & ND & ND & Survived \\
\hline 10 & Pos & ND & Neg & Pos & 27 & MTB & $\mathrm{S}$ & $\mathrm{S}$ & Survived \\
\hline 11 & Pos & ND & Neg & Neg & 20 & ND & ND & ND & Survived \\
\hline 12 & Pos & ND & Neg & $\mathrm{Neg}$ & 24 & MTB & $\mathrm{S}$ & $\mathrm{S}$ & Survived \\
\hline 13 & Pos & ND & ND & ND & 24 & MTB & $\mathrm{S}$ & $\mathrm{S}$ & Died \\
\hline 14 & Pos & ND & Neg & Neg & 27 & MTB & $\mathrm{S}$ & $\mathrm{R}$ & Unknown \\
\hline
\end{tabular}

*Time to positivity of TB blood cultures;; MTB: M. tuberculosis; ND: not done; R: resistant; S: susceptible 
also reported that in HIV patients with TB, a considerable proportion of sputum samples are negative for AFB $[8,9]$. One study compared AFB smears with mycobacterial culture and showed that AFB smears on three sputa failed to diagnose TB in $63 \%$ of HIV-infected patients [5]. However, another study showed that the best first step of TB diagnosis is still to perform AFB smear on at least 2 sputum specimens and if the smears are negative and TB is still suspected, sputum should be cultured [5]. Therefore it is essential also to culture sputum specimens in HIV-infected patients and not solely rely on AFB smear. This is a limitation of our study because sputum culture was generally not performed for various reasons, including: the patient cannot expectorate or was not requested by the doctor as sputum culture takes a long time and results are usually available after discharge. Blood cultures may also yield fungi, like $P$. marneffei, and therefore produce other relevant results for patient management.

In this study, HIV-infected patients with mycobacteremia are likely to have lower CD4 count than HIV-infected patients without mycobacteremia. M.tuberculosis was the most common cause of bloodstream infection (BSI) in HIV-infected cases in our study. This is consistent with other studies reporting an association with low CD4 counts and BSI [18]. One study conducted in 3 countries including Vietnam observed that HIV-infected patients have a high likelihood of BSI, particularly if their CD4 count $<100$ cells $/ \mathrm{mm}^{3}[18]$. In our population, the majority $(80 \%)$ of the patients had CD 4 counts $<100$ cells $/ \mathrm{mm}^{3}$ as our study generally concerns HIV-infected patients who are admitted with opportunistic infections (AIDS defining illness). Mycobacteremia cases generally occurred in those not yet on ART. One mycobacteremia case occurred in a patients 2 months on ART but with remaining low CD4 counts, illustrating treatment failure for unknown reasons. Of the four cases that died in this study, one did not receive any $\mathrm{TB}$ treatment and the other three died one day after starting TB treatment. One lethal case was infected with MDR TB and one with a rifampicin resistant TB. Late installment of TB treatment was likely the cause of death in these fatal cases.

Mycobacteria were the most commonly found pathogens in the blood cultures in this study population, followed by $P$. marneffei. These results are consistent with a similar Asian study, where Myco/F lytic blood cultures were performed on HIV patients [18]. This study found that M. tuberculosis was the most frequent isolated pathogen in HIV patients, followed by Cryptococcus species and P. marneffei.

In resource constrained settings like Vietnam, mycobacterial blood culture is generally not performed. Hence, we believe our data on mycobacterial blood cultures can contribute to improve TB diagnosis in endemic TB countries in Asia, particularly in HIV-infected patients. Although many studies have different results regarding mycobacterial blood cultures, they could not negate the usefulness of blood cultures in helping to diagnose $\mathrm{TB}$, especially when other specimens are difficult to collect or unavailable $[18,19]$. The yield of this method may be increased if more than one blood specimen is collected. However, costs and time to detection are high and therefore is generally not considered feasible.

\section{Conclusion}

Blood cultures for TB can be useful for TB diagnosis, particularly in immunosuppressed HIV patients when sputum or other specimens cannot be obtained. However, mycobacterial blood culture has some important limitations, including the long incubation time, costs, and sensitivity. mycobacterial blood cultures cannot replace sputum AFB smear and sputum culture, but is a valuable additional method to combine with other basic methods for better yield of TB testing. Methods to shorten time to detection of mycobacteria in the blood would improve the clinical utility.

\section{Acknowledgements}

The MycoF/Lytic Bactec bottles were made available free of charge by Becton Dickinson upon our request and initiative. The sponsors had no influence on the trial design, or the decision to write a report and submission for publication.

\section{Author's contributions}

NTND and HW wrote the manuscript and analyzed the data. HW, NVT, PVC and NVK designed the study. NTND, DTT and NTL participated in the collection of data. PH participated to analyze data. NTND and DTT participated labolatory testing. All authors have read and approved the final manuscript.

\section{References}

1. World Health Organization (2013) Global Tuberculosis Control 2013. World Health Organization Report WHO/HTM/TB/20135. Available at http://apps.who.int/iris/bitstream/10665/91355/1/9789241564 656_eng.pdf. Accessed April 2013. 
2. Parsons LM, Somoskovi A, Gutierrez C, Lee E, Paramasivan $\mathrm{CN}$, Abimiku A, Spector S, Roscigno G, Nkengasong J (2011) Laboratory diagnosis of tuberculosis in resource-poor countries: challenges and opportunities. Clin Microbiol Rev 24: 314-350.

3. Perkins MD, Cunningham J (2007) Facing the crisis: improving the diagnosis of tuberculosis in the HIV era. J Infect Dis 196 Suppl 1: S15-27.

4. Archibald LK, den Dulk MO, Pallangyo KJ, Reller LB (1998) Fatal Mycobacterium tuberculosis bloodstream infections in febrile hospitalized adults in Dar es Salaam, Tanzania. Clin Infect Dis 26: 290-296.

5. Monkongdee P, McCarthy KD, Cain KP, Tasaneeyapan T, Nguyen HD, Nguyen TN, Nguyen TB, Teeratakulpisarn N, Udomsantisuk N, Heilig C, Varma JK (2009) Yield of acidfast smear and mycobacterial culture for tuberculosis diagnosis in people with human immunodeficiency virus. Am J Respir Crit Care Med 180: 903-908.

6. Mendelson M (2007) Diagnosing tuberculosis in HIVinfected patients: challenges and future prospects. $\mathrm{Br}$ Med Bull 81-82: 149-165.

7. Cain KP, McCarthy KD, Heilig CM, Monkongdee P, Tasaneeyapan T, Kanara N, Kimerling ME, Chheng P, Thai S, Sar B, Phanuphak P, Teeratakulpisarn N, Phanuphak N, Nguyen HD, Hoang TQ, Le HT, Varma JK (2010) An algorithm for tuberculosis screening and diagnosis in people with HIV. N Engl J Med 362: 707-716.

8. Elliott AM, Namaambo K, Allen BW, Luo N, Hayes RJ, Pobee JO, McAdam KP (1993) Negative sputum smear results in HIV-positive patients with pulmonary tuberculosis in Lusaka, Zambia. Tuber Lung Dis 74: 191-194.

9. Getahun H, Harrington M, O'Brien R, Nunn P (2007) Diagnosis of smear-negative pulmonary tuberculosis in people with HIV infection or AIDS in resource-constrained settings: informing urgent policy changes. Lancet 369: 20422049.

10. Hillemann D, Weizenegger M, Kubica T, Richter E, Niemann $S$ (2005) Use of the genotype MTBDR assay for rapid detection of rifampin and isoniazid resistance in Mycobacterium tuberculosis complex isolates. J Clin Microbiol 43: 3699-3703.

11. Golden MP, Vikram HR (2005) Extrapulmonary tuberculosis: an overview. Am Fam Physician 72: 1761-1768.

12. Elder NC (1992) Extrapulmonary tuberculosis. A review. Arch Fam Med 1: 91-98.
13. Grinsztejn B, Fandinho FC, Veloso VG, Joao EC, Lourenco MC, Nogueira SA, Fonseca LS, Werneck-Barroso E (1997) Mycobacteremia in patients with the acquired immunodeficiency syndrome. Arch Intern Med 157: 23592363.

14. Bouza E, Diaz-Lopez MD, Moreno S, Bernaldo de Quiros JC, Vicente T, Berenguer J (1993) Mycobacterium tuberculosis bacteremia in patients with and without human immunodeficiency virus infection. Arch Intern Me 153: 496500.

15. Weyer K, Kantor IND, Kim SJ, Frieden T, Laszlo A, Luelmo F, Norval P-Y, Rieder H, Valenzuela P Laboratory Services in Tuberculosis Control: Part II: Microscopy: World Health Organization, Geneva, Switzerland 1998.

16. Kulski JK, Khinsoe C, Pryce T, Christiansen K (1995) Use of a multiplex PCR to detect and identify Mycobacterium avium and $\mathrm{M}$. intracellulare in blood culture fluids of AIDS patients. J Clin Microbiol 33: 668-674.

17. Ben Kahla I, Ben Selma W, Marzouk M, Ferjeni A, Ghezal S, Boukadida J (2011) Evaluation of a simplified IS6110 PCR for the rapid diagnosis of Mycobacterium tuberculosis in an area with high tuberculosis incidence. Pathol Biol (Paris) 59: $161-165$

18. Varma JK, McCarthy KD, Tasaneeyapan T, Monkongdee P, Kimerling ME, Buntheoun E, Sculier D, Keo C, Phanuphak P, Teeratakulpisarn N, Udomsantisuk N, Dung NH, Lan NT, Yen NT, Cain KP (2010) Bloodstream infections among HIVinfected outpatients, Southeast Asia. Emerg Infect Dis 16: 1569-1575.

19. Heysell SK, Thomas TA, Gandhi NR, Moll AP, Eksteen FJ, Coovadia Y, Roux L, Babaria P, Lalloo U, Friedland G, Shah S (2010) Blood cultures for the diagnosis of multidrugresistant and extensively drug-resistant tuberculosis among HIV-infected patients from rural South Africa: a crosssectional study. BMC Infect Dis 10: 344.

\section{Corresponding author}

Heiman Wertheim

Oxford University Clinical Research Unit, National Hospital for Tropical Diseases, 78 GiaiPhong Street, Hanoi, Vietnam.

Phone: +84435764320

Fax: +84435764320

Email: heiman.wertheim@gmail.com

Conflict of interests: No conflict of interests is declared. 